Original Article

\title{
Comparison of Mitral Valve Repair versus Replacement for the Progression of Functional Tricuspid Regurgitation
}

\author{
Yang Gao, MD, ${ }^{1}$ Shubin Li, MD,${ }^{2}$ Xijing Zhuang, MD,${ }^{3}$ Feng Gao, MD,${ }^{3}$ Lei Shi, MD, ${ }^{3}$ \\ and $\mathrm{Xu}$ Meng, $\mathrm{MD}^{1}$
}

\begin{abstract}
Background: Function tricuspid regurgitation (TR) is frequently observed in patients undergoing mitral valve surgery. It is unclear that mitral valve repair (MVr) or mitral valve replacement (MVR) has influence on the likelihood of late TR progression.

Methods: This study included 193 patients with degenerative mitral valve disease who underwent either MVr or MVR. Detailed preoperative materials, follow-up information, and echocardiographic data were collected and statistically analyzed.

Results: At 6 and 12 months postoperatively, MVR patients were more likely to have New York Heart Association (NYHA) class III or IV symptoms than MVr patients (6 mo: $15.2 \%$ vs $5.0 \%, 12 \mathrm{mo:} 13.0 \%$ vs $4.0 \%$, both $P<0.05$ ). At 24 months, the incidence of Grade 1+ TR was significantly higher in MVR patients than MVr patients $(25.0 \%$ vs $12.9 \%, P<0.05)$. In univariate analysis, age (odds ratio $[\mathrm{OR}]=1.036, P=0.036)$, MVR $(\mathrm{OR}=\mathbf{2 . 2 5 6}, P=\mathbf{0 . 0 3 3})$, and preoperative $\mathrm{TR}$ area $(\mathrm{TRA} ; \mathrm{OR}=1.541, P=0.047)$ were significant predictors for TR progression. In multivariate logistics analysis, only MVR was independently risk factor $(P=\mathbf{0 . 0 0 6})$. Subsequently, patients were divided into tricuspid valve repair (TVr) group and untreated group. In both subgroups, MVR patients were associated with significantly larger TRA $(P<0.01)$.

Conclusion: MVR was an independent risk factor for TR progression, whether tricuspid valve was treated or not.
\end{abstract}

Keywords: mitral valve repair, replacement, functional tricuspid valve regurgitation, risk factor

\begin{abstract}
${ }^{1}$ Department of Cardiology Surgery, Beijing Anzhen Hospital, Capital Medical University, Beijing, China

${ }^{2}$ Department of Cardiology Surgery, Xinxiang Central Hospital, Xinxiang, China

${ }^{3}$ Department of Cardiology Surgery, Dalian Municipal Central Hospital, Dalian, China
\end{abstract}

Received: May 10, 2019; Accepted: July 16, 2019

Corresponding author: Xu Meng, MD. Department of Cardiology Surgery, No. 9 Ward of Cardiac-Valve Surgery \& Heart Transplantation Center, Capital Medical University Affliated Beijing Anzhen Hospital, No. 2 Anzhen Road, Chaoyang District, Beijing, 100029, China

Email: asdasd1234512@126.com

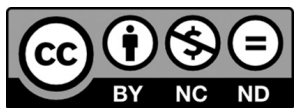

This work is licensed under a Creative Commons Attribution-NonCommercialNoDerivatives International License.

(C2020 The Editorial Committee of Annals of Thoracic and Cardiovascular Surgery

\section{Introduction}

Degenerative mitral valve disease is the most prevalent heart valvular disease, which affects about $2 \%$ of population worldwide. ${ }^{1,2)}$ Leaflet prolapse, combined with varying degree of mitral valve regurgitation, occurred in most of these patients due to the elongation and rupture of chordae tendineae. ${ }^{3)}$ In such situation, surgical correction is always the optimal choice. There are mainly two procedures, mitral valve repair (MVr) and mitral valve replacement (MVR), each of them has its unique advantages. MVr confers advantages of preservation of left ventricular function, avoidance of long-term anticoagulants and greater freedoms from endocarditis, ${ }^{4)}$ whereas MVR has achieved to decrease its surgical risk by preservation techniques which protect left ventricular geometry and function. ${ }^{5)}$ 
Function tricuspid regurgitation (TR), secondary to tricuspid annular dilatation, is commonly present in patients with degenerative mitral valve disease. For patients undergoing mitral valve surgery, late functional TR progression is frequently observed in the follow-up. The development of moderate-to-severe TR was associated with a poor prognosis.6) Thus, extensive studies were performed to evaluate the risk factors for late TR progression, such as age, female gender, atrial fibrillation, left ventricular dysfunction, and tricuspid annular diameter index. ${ }^{7-9)}$ However, there were scare evidence that investigate different surgical procedure, MVR or MVr, that affects the likelihood of TR progression. The present study aims to compare the influence of MVR versus MVr on the late functional TR development, based on mid-term follow-up and echocardiographic data in MVR or MVr patients with preoperative no to trivial TR.

\section{Methods}

\section{Subjects}

From January 2013 to June 2015, 193 consecutive patients who underwent surgical correction of degenerative mitral valve regurgitation, receiving procedure of either MVR or MVr, at Cardiac Surgery Department of Capital medical university affiliated Beijing Anzhen Hospital, were retrospectively analyzed. Preoperative functional TR in all patients were lower than Grade 1. Functional TR was defined as TR with absence of anatomic malformation of tricuspid valvular leaflets or sub-valvular structures. We quantified TR based on echocardiographic results as insignificant (Grade 1), mild (Grade 2), moderate (Grade 3), and moderately severe and severe (Grade 4). All patients underwent initial procedure of isolated mitral valve surgery or concomitant tricuspid valve repair (TVr).

Exclusion criteria were patients undergoing concomitant coronary artery bypass graft surgery, aortic valve surgery, or surgical ablation of atrial fibrillation. Patients coexisted with congenital heart disease, myocardiopathies, or infective endocarditis were also excluded. Moreover, we excluded patients with incomplete clinical data and re-operative patients.

This study was performed in accordance with Declaration of Helsinki Principles revised in 1983. All participants provided written informed consent. The study was ethically approved by institutional review committee of Beijing Anzhen Hospital.

\section{Interventions}

A full median sternotomy was performed in each operation. The operation was conducted under a standard cardiopulmonary bypass established by ascending aortic and both venae cavae. Moderate hypothermia and warm blood cardioplegia for myocardial protection were used. Surgical approach was either through right atrium or the interatrial groove. For MVr, several surgical techniques were used. For patients with posterior leaflet prolapse, we commonly use quadrangular resection or folding valvuloplasty. In several posterior leaflet prolapses patients, we also use artificial chordae. For patients with anterior leaflet prolapse with or without ruptured chordae, the surgical techniques included artificial chordae, chordal shortening, and chordal transposition. For patients with commissure regurgitation, we use commissure fusion technique. A ring annuloplasty was performed in all MVr patients. According to surgeons' preference, either a rigid-ring such as Meditronic Duran AnCore ring or a flexible-ring such as Carpentier-Edwards Physio was performed. The ring size was determined by measuring the area of anterior leaflet with prosthetic sizers provided by its manufacturers. For MVR, all patients received posterior leaflet preservation technique. Among these, 76 implanted mechanical prosthetic valves and 17 bioprosthetic valves were performed. According to surgeons' decision, concomitant TVr was conducted by ring annuloplasty using prosthesis.

\section{Echocardiography}

We routinely utilized intraoperative transesophageal echocardiography (TEE) in all patients; and transthoracic echocardiography (TTE) was performed at $6,12,18$, and 24 months during the follow-up. The details for echocardiographic measurements in routine clinical practice were described in our previous studies. ${ }^{10,11)}$

\section{Statistical analysis}

Continuous variables were expressed as means \pm standard deviation (SD). Discrete data were presented as percentages. Student's T-test was performed to compare continuous variables between two groups. For comparison of categorical data, $\chi^{2}$ or Fisher's exact tests were used. We conducted both univariate and binary multivariate logistic regression models to assess the risk factors influencing progression of TR. Reported $P$ values were all two-tailed, and $P$ value $\leq 0.05$ was considered as statistically significance. We used SPSS Statistics 19.0 software (IBM, Armonk, NY, USA) for data analysis. 
Table 1 Clinical characteristics of patients

\begin{tabular}{|c|c|c|c|c|}
\hline Variables & All subjects $(\mathrm{n}=193)$ & $\operatorname{MVR}(\mathrm{n}=92)$ & $\operatorname{MVr}(\mathrm{n}=101)$ & $P$ value \\
\hline \multicolumn{5}{|l|}{ Demographic data } \\
\hline Age (years) & $51.8 \pm 12.1$ & $54.7 \pm 11.0$ & $49.1 \pm 12.5$ & 0.397 \\
\hline Sex & & & & 0.535 \\
\hline Male & $126(65.3)$ & $58(63)$ & $68(67.3)$ & \\
\hline Female & $67(34.7)$ & $34(37)$ & $33(32.7)$ & \\
\hline \multicolumn{5}{|l|}{ Symptoms and intervention } \\
\hline NYHA class & & & & 0.453 \\
\hline I-II & $131(67.9)$ & $60(65.2)$ & $71(70.2)$ & \\
\hline III-IV & $62(32.1)$ & $32(34.8)$ & $30(29.8)$ & \\
\hline Concomitant TVr & $113(58.5)$ & $54(58.7)$ & $59(58.4)$ & 0.969 \\
\hline \multicolumn{5}{|l|}{ Echocardiographic data } \\
\hline $\mathrm{EF}(\%)$ & $61.2 \pm 7.7$ & $59.9 \pm 7.8$ & $62.3 \pm 7.4$ & 0.469 \\
\hline E/A ratio & $1.70 \pm 0.66$ & $1.77 \pm 0.69$ & $1.64 \pm 0.63$ & 0.185 \\
\hline FAC $(\%)$ & $33.3 \pm 5.69$ & $32.2 \pm 5.83$ & $34.4 \pm 5.37$ & 0.333 \\
\hline LVEDD (mm) & $58.2 \pm 8.0$ & $59.6 \pm 8.7$ & $56.9 \pm 7.0$ & 0.039 \\
\hline LVESD (mm) & $38.3 \pm 6.8$ & $39.9 \pm 7.4$ & $37.0 \pm 5.9$ & 0.030 \\
\hline $\mathrm{RV}(\mathrm{mm})$ & $21.79 \pm 3.75$ & $32.21 \pm 5.83$ & $34.36 \pm 5.37$ & 0.064 \\
\hline Preoperative-MR & & & & 0.958 \\
\hline Grade 3 & $17(8.8)$ & $8(8.6)$ & $9(8.9)$ & \\
\hline Grade 4 & $176(91.2)$ & $84(91.3)$ & $92(91.1)$ & \\
\hline Preoperative-TR & & & & 0.028 \\
\hline No TR & $49(25.4)$ & $30(32.6)$ & $19(18.8)$ & \\
\hline Grade 1 & $144(74.6)$ & $62(67.4)$ & $82(81.2)$ & \\
\hline Tricuspid regurgitation area $\left(\mathrm{cm}^{2}\right)$ & $1.8 \pm 1.6$ & $1.6 \pm 0.9$ & $2.0 \pm 2.0$ & 0.987 \\
\hline Mechanical valve (n, \%) & $75(81.5 \%)$ & & & \\
\hline $27 \mathrm{~mm}$ & 28 & & & \\
\hline $29 \mathrm{~mm}$ & 45 & & & \\
\hline $31 \mathrm{~mm}$ & 2 & & & \\
\hline Bioprosthetic valve (n, \%) & $17(18.5 \%)$ & & & \\
\hline $27 \mathrm{~mm}$ & 6 & & & \\
\hline $29 \mathrm{~mm}$ & 10 & & & \\
\hline $31 \mathrm{~mm}$ & 1 & & & \\
\hline Physio ring (n, \%) & & $28(27.7 \%)$ & & \\
\hline $28 \mathrm{~mm}$ & & 2 & & \\
\hline $30 \mathrm{~mm}$ & & 11 & & \\
\hline $32 \mathrm{~mm}$ & & 14 & & \\
\hline $34 \mathrm{~mm}$ & & 1 & & \\
\hline Physio II ring (n, \%) & & $49(48.5 \%)$ & & \\
\hline $28 \mathrm{~mm}$ & & 7 & & \\
\hline $30 \mathrm{~mm}$ & & 19 & & \\
\hline $32 \mathrm{~mm}$ & & 23 & & \\
\hline Duran ring (n, \%) & & $24(23.8 \%)$ & & \\
\hline $27 \mathrm{~mm}$ & & 5 & & \\
\hline $29 \mathrm{~mm}$ & & 10 & & \\
\hline $31 \mathrm{~mm}$ & & 8 & & \\
\hline $33 \mathrm{~mm}$ & & 1 & & \\
\hline
\end{tabular}

NYHA: New York Heart Association; TVr: tricuspid valve repair; EF: ejection fraction; E/A ratio: the ratio of the early diastolic filling to peak atrial filling velocities; FAC\%: the fractional area change; LVEDD: left ventricular end diastolic diameter; LVESD: left ventricular end systolic diameter; RV: right ventricular diameter; MR: mitral regurgitation; MVr: mitral valve repair; MVR: mitral valve replacement; TR: tricuspid regurgitation 
Table 2 Comparison of TR grade and NYHA symptoms between two groups

\begin{tabular}{llcc}
\hline Follow-up & MVR & MVr & $P$ value \\
\hline 6 months & & & \\
TR Grade 0-1 & $80(87)$ & $94(93.1)$ & 0.156 \\
TR Grade 2-4 & $12(13)$ & $7(6.9)$ & \\
NYHA class I-II & $78(84.8)$ & $96(95)$ & 0.017 \\
NYHA class III-IV & $14(15.2)$ & $5(5)$ & \\
12 months & & & \\
TR Grade 0-1 & $78(87)$ & $91(93.1)$ & 0.266 \\
TR Grade 2-4 & $14(13)$ & $10(6.9)$ & \\
NYHA class I-II & $79(85.9)$ & $93(92.1)$ & 0.168 \\
NYHA class III-IV & $13(14.1)$ & $8(7.9)$ & \\
18 months & & & \\
TR Grade 0-1 & $76(87)$ & $89(93.1)$ & 0.280 \\
TR Grade 2-4 & $16(13)$ & $12(6.9)$ & \\
NYHA class I-II & $75(81.5)$ & $90(89.1)$ & 0.136 \\
NYHA class III-IV & $17(18.5)$ & $11(10.9)$ & \\
24 months & & & \\
TR Grade 0-1 & $69(75)$ & $88(87.1)$ & 0.031 \\
TR Grade 2-4 & $23(25)$ & $13(12.9)$ & \\
NYHA class I-II & $80(87)$ & $97(96)$ & 0.022 \\
NYHA class III-IV & $12(13)$ & $4(4)$ & \\
\hline
\end{tabular}

FTR: functional tricuspid regurgitation; MVr: mitral valve repair; MVR: mitral valve replacement; NYHA: New York Heart Association; TR: tricuspid regurgitation

\section{Results}

Totally, 92 patients (47.7\%) underwent MVR and 101 (52.3\%) underwent MVr. The average age of all participants was $51.8 \pm 12.1$ years. There were 126 males $(65.3 \%)$ and 67 females (34.7\%). Patients' preoperative details are presented in Table 1. A significantly higher left ventricular end diastolic diameter (LVEED; $59.6 \pm$ 8.7 vs $56.9 \pm 7.0, P<0.05)$ and left ventricular end systolic diameter (LVESD; $39.9 \pm 7.4$ vs $37.0 \pm 5.9, P<0.05$ ) was observed in MVR group compared with MVr group. Nevertheless, patients in MVr group were more likely to have a Grade 1 TR compared with patients in MVR group $(81.2 \%$ vs $67.4, P<0.05)$. In addition, 75 patients received a mechanical valve $(81.5 \%)$. The sizes of the artificial valves and the ring are listed in Table 2.

We assessed functional TR and New York Heart Association (NYHA) symptoms at 6, 12, 18, and 24 months postoperatively, as presented in Table 3. All included participants completed 2-year echocardiographic follow-up. At 6 months, MVR patients were more likely to have NYHA class III or IV symptoms ( $15.2 \%$ vs $5.0 \%, P<0.05)$. But no significances were observed at 12 and 18 months (all $P>0.05$ ). There was a significant difference of NYHA symptoms at 24 months, as 13\% patients in MVR group were more likely to have NYHA class III or IV symptoms compared with $4 \%$ patients in $\mathrm{MVr}$ group $(P<0.05)$.

We did not observe a significant difference of TR Grade between two groups at 6,12 , and 18 months. However, the incidence of Grade 1+ TR was significantly higher in MVR group compared with patients in MVr group at 24 months ( $25 \%$ vs $12.9 \%, P<0.05)$.

The predictors of mid-term Grade 1+ TR were age (odds ratio $[\mathrm{OR}]=1.036, P=0.036)$, $\mathrm{MVR}(\mathrm{OR}=2.256$, $P=0.033)$, and preoperative tricuspid regurgitation area (TRA) measured by TTE (OR $=1.541, P=0.047)$, as listed in Table 3. Patients undergoing MVR were 2.26 times more likely to experience Grade 1+ TR at 24 months than patients undergoing MVr. Notably, concomitant $\mathrm{TVr}$ procedure was not a significant univariate predictor for TR progression (Table 4). In multivariate regression analysis, only MVR was independently significant factor affecting mid-term progression of TR $(P=0.006)$.

In subgroup analysis, we consider $\mathrm{TVr}$ as a critical confounder that affects the comparison between MVR and MVr group (although TVr was not a predictor of TR progression). We divided patients into two groups: $\mathrm{TVr}$ group $(n=113)$ and tricuspid untreated group $(n=80)$. In either group, we compared the TRA between MVR and MVr group. As shown in Fig. 1, it is suggested that MVR patients were associated with a significantly larger TRA than MVr patients at 24 months (all $P<0.01$ ), whether tricuspid valve was treated or not.

To explore the mechanism that MVr was better than MVR for late TR, we further compared echocardiography parameters such as ejection fraction $(\mathrm{EF})$, the ratio of the early diastolic filling to peak atrial filling velocities (E/A ratio), the fractional area change (FAC\%), LVEDD, LVESD, and right ventricular diameter (RV). As shown in Table 4, at 24 months of follow-up, patients in MVr group were associated with a higher EF and FAC, and a lower LVEDD, LVESD, and RV (all $P<0.05$ ).

\section{Discussion}

Functional TR was frequent in patients with mitral valve surgery, which has a substantial impact on quality of life and mortality. ${ }^{12,13)}$ Extensive studies explored the risk factors of TR development after mitral valve surgery, but lack of evidence address the influence of different surgery approach, MVR or MVr, on the likelihood of TR progression. The present study confirmed that MVR was an independent risk factor that affect late TR progression. Patients undergoing MVR procedure were 2.26 times 
Table 3 Risk factors for late TR progression at 24 months of follow-up

\begin{tabular}{lll}
\hline Risk factor & OR $(95 \%$ CI $)$ & $P$ value \\
\hline Age & $1.036(1.002-1.072)$ & 0.036 \\
Female gender & $1.915(0.918-3.995)$ & 0.083 \\
MVR & $2.256(1.066-4.775)$ & 0.033 \\
Preoperative NYHA classification & $1.070(0.495-2.311)$ & 0.863 \\
Preoperative EF & $1.002(0.956-1.051)$ & 0.927 \\
Preoperative MR Grade & $3.972(0.509-30.972)$ & 0.188 \\
Preoperative TR Grade & $1.886(0.734-4.848)$ & 0.188 \\
Preoperative tricuspid regurgitation area & $1.541(1.006-2.360)$ & 0.047 \\
Preoperative LVEDD & $0.982(0.938-1.028)$ & 0.437 \\
Preoperative LVESD & $1.000(0.948-1.055)$ & 0.989 \\
Preoperative LA size & $1.010(0.969-1.053)$ & 0.635 \\
Preoperative RA size & $1.001(0.954-1.050)$ & 0.975 \\
Preoperative RV size & $1.015(0.926-1.113)$ & 0.753 \\
Preoperative systolic PAP & $1.008(0.989-1.027)$ & 0.413 \\
Concomitant tricuspid valve repair & $0.749(0.362-1.551)$ & 0.437 \\
\hline
\end{tabular}

EF: ejection fraction; LA: left atrium; LVEDD: left ventricular end diastolic diameter; LVESD: left ventricular end systolic diameter; MR: mitral regurgitation; NYHA: New York Heart Association; PAP: pulmonary artery pressure; RA: right atrium; RV: right ventricle; TR: tricuspid regurgitation
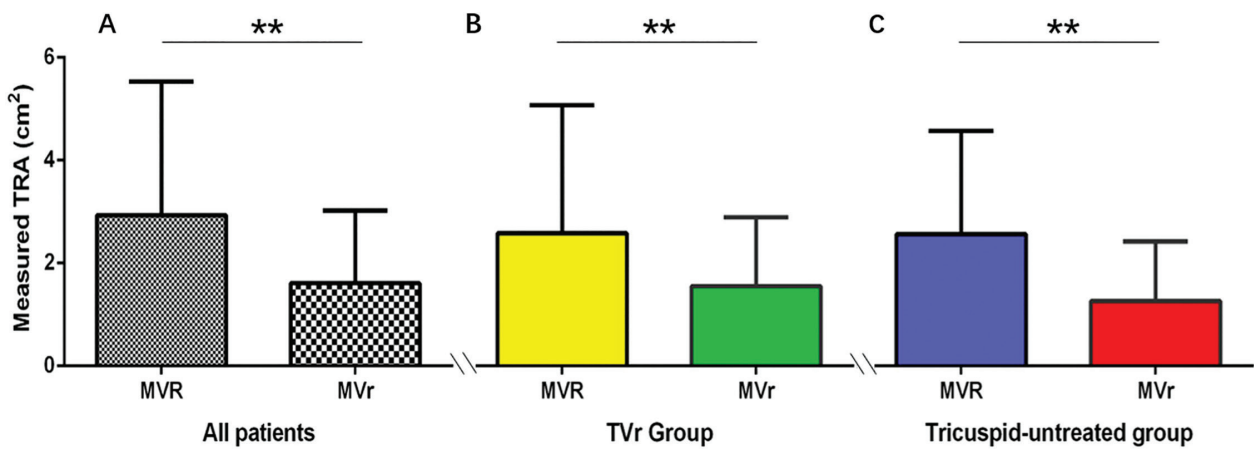

Fig. 1 Subgroup analysis to compare tricuspid regurgitation area between MVR and MVr group. (A) The whole cohort; (B) tricuspid valve repair group; (C) tricuspid valve untreated group. MVr: mitral valve repair; MVR: mitral valve replacement

more likely to experience a Grade 1+ TR development than patients with MVr surgery. In addition, the results show that older age and preoperative TRA were also univariate predictors for TR progression. However, for patients with a minimal TR, TVr procedure did not have a significant influence on late TR progression.

Our results indicated that compared with MVR surgery, $\mathrm{MVr}$ is more effective in control of TR progression. Previous study by Rajbanshi BG et al. ${ }^{9)}$ reported that MVR was not a significant risk factor for functional TR development at 5 years of follow-up. However, preoperative Grade 1+ patients included in his study account for a large proportion, which interfered the observation of postoperative TR progression. Here, we recruited patients with preoperative no to trivial TR, which ensure a more homogenous group. Notably, our results were consistent with the results reported by Fukunaga et al. ${ }^{14)}$ Their study demonstrated that MVR was a significant risk factor for residual TR (moderate to severe). They concluded that MVR patients had a higher incidence of atrial fibrillation and a higher systolic pulmonary artery pressure (SPAP), which were associated with higher likelihood of TR progression. However, patients in Fukunaga's study underwent mitral valve surgery mainly for rheumatic etiology, which were not directly applicable to patients with degenerative mitral valve disease. A study by Angeloni et al. found that prosthesis-patient mismatch significantly 
Table 4 Comparison of echocardiography parameters between two groups

\begin{tabular}{lcrr}
\hline Variables & MVR group & MVr group & $P$ value \\
\hline EF $(\%)$ & $56.2 \pm 10.0$ & $63.3 \pm 5.9$ & $<0.001$ \\
E/A ratio & $1.8 \pm 4.4$ & $1.2 \pm 0.4$ & 0.202 \\
FAC $(\%)$ & $30.6 \pm 6.1$ & $35.3 \pm 5.0$ & $<0.001$ \\
LVEDD $(\mathrm{mm})$ & $56.7 \pm 6.9$ & $46.7 \pm 5.6$ & $<0.001$ \\
LVESD $(\mathrm{mm})$ & $37.5 \pm 7.0$ & $30.7 \pm 4.8$ & $<0.001$ \\
RV $(\mathrm{mm})$ & $22.7 \pm 3.5$ & $21.3 \pm 3.3$ & 0.005 \\
\hline
\end{tabular}

EF: ejection fraction; E/A ratio: the ratio of the early diastolic filling to peak atrial filling velocities; FAC\%: the fractional area change; LVEDD: left ventricular end diastolic diameter; LVESD: left ventricular end systolic diameter; MVr: mitral valve repair; MVR: mitral valve replacement; $\mathrm{RV}$ : right ventricle

affects late TR progression in patients undergoing MVR. ${ }^{15)}$ Another study by Grapsa ${ }^{16)}$ showed that MVr procedure resulted in more favorable reverse $\mathrm{RV}$ remodeling, which had beneficial impact on TR control. The two reasons mentioned above can explain results of our study that MVR had an inferior impact on late TR progression. In our study, we analyzed that patients in MVr group were associated with significantly higher EF and FAC, and lower LVEDD, LVESD, and RV at 24 months of follow-up, which indicated that MVr patients showed better LV and RV function than MVR patients, which had beneficial impact on TR control.

The present study also indicated that age and preoperative TRA were predictors for TR progression. The impact of age on TR progression was previously investigated. A study by Hata et al. ${ }^{17)}$ revealed that older age was independent risk factor of recurrent moderate-to-severe TR. Kusajima et al. ${ }^{7)}$ reported that age has an independent but minor impact on TR progression, with an OR of 1.1. Similarly, the effect of age was minimal in our study, with an OR of 1.036. In addition, our study demonstrated that measured TRA, which quantificationally reflects preoperative TR severity, also independently and significantly affect mid-term TR progression, with an OR of 1.541. The results suggest of accurately echocardiographic measurement of relevant parameters that reflect TR severity before surgery. Moreover, several studies showed that female gender was a risk factor for TR progression. ${ }^{9}{ }^{, 18)}$ In the present study, we also found that females were 1.915 times more likely to develop to a Grade 1+ TR, but the results were insignificant $(P=0.083)$.

In our institution, whether prophylactic TVr was performed or not was mainly determined by the extent of tricuspid annulus dilatation, which was consistent with previously published studies. ${ }^{19,20)}$ In our study, prosthetic ring was performed in all TVr operations. In many studies, $\mathrm{TVr}$ with a prosthetic ring showed superior results than non-ring annuloplasty. ${ }^{21,22)}$ Considering that the treatment of tricuspid valve was an important confounder, we performed a subgroup analysis by dividing patients into TVr group and tricuspid untreated group. The analysis demonstrated that postoperative TRA, which was measured by echocardiography to reflect TR severity, was significantly larger in MVR group than that of MVr group in both subgroups.

Notably, the homogenous population was the strength of this study. We recruited patients with degenerative mitral valve disease and coexisting with preoperative no to trivial functional TR, which eliminated the confounders of (1) the etiology (rheumatic, congenital, ischemic) of mitral valve disease and (2) preoperatively developed TR.

The study has several limitations. First, the study was limited by its retrospective nature, which has a relative low quality of evidence. Second, to perform a perfect comparison, this study screened the data and only included patients with a complete follow-up and echocardiographic information, and ruled out the lost and dead patients, which resulted in considerable report bias. Third, our study was a mid-term report, further study should be conducted to demonstrate the long-term results of MVR versus MVr for late TR progression.

\section{Conclusion}

The present study confirmed that MVR was an independent risk factor for late TR progression. Older age and preoperative TRA were also significant predictors. Besides, the study found that surgical correction of tricuspid valve was not a factor that influence postoperative TR progression, in patients with preoperative no to trivial TR. Further high-quality and prospective studies are required to confirm our findings.

\section{Disclosure Statement}

All the authors declare that there are no competing interests.

\section{References}

1) Enriquez-Sarano M, Akins CW, et al. Mitral regurgitation. Lancet 2009; 373: 1382-94.

2) Lazam S, Vanoverschelde JL, Tribouilloy $C$, et al. Twenty-year outcome after mitral repair versus replacement for severe degenerative mitral regurgitation: analysis 
of a large, prospective, multicenter, international registry. Circulation 2017; 135: 410-22.

3) Adams DH, Rosenhek R, Falk V. Degenerative mitral valve regurgitation: best practice revolution. Eur Heart J 2010; 31: 1958-66.

4) Gillinov AM, Blackstone EH, Nowicki ER, et al. Valve repair versus valve replacement for degenerative mitral valve disease. J Thorac Cardiovasc Surg 2008; 135: 885-93, 893.e1-2.

5) Sénéchal M, MacHaalany J, Bertrand OF, et al. Predictors of left ventricular remodeling after surgical repair or replacement for pure severe mitral regurgitation caused by leaflet prolapse. Am J Cardiol 2013; 112: 567-73.

6) Shiran A, Sagie A. Tricuspid regurgitation in mitral valve disease incidence, prognostic implications, mechanism, and management. J Am Coll Cardiol 2009; 53: 401-8.

7) Kusajima K, Fujita T, Hata H, et al. Long-term echocardiographic follow-up of untreated $2+$ functional tricuspid regurgitation in patients undergoing mitral valve surgery. Interact Cardiovasc Thorac Surg 2016; 23: $96-103$.

8) Vaturi M, Kotler T, Shapira Y, et al. Prevalence of late functional tricuspid regurgitation in degenerative mitral regurgitation surgery. J Heart Valve Dis 2016; 25: 157-61.

9) Rajbanshi BG, Suri RM, Nkomo VT, et al. Influence of mitral valve repair versus replacement on the development of late functional tricuspid regurgitation. J Thorac Cardiovasc Surg 2014; 148: 1957-62.

10) Wei D, Han J, Zhang H, et al. The correlation between the coaptation height of mitral valve and mitral regurgitation after mitral valve repair. J Cardiothorac Surg 2017; 12: 120.

11) Fu JT, Popal MS, Jiao YQ, et al. A predictor for mitral valve repair in patient with rheumatic heart disease: the bending angle of anterior mitral leaflet. J Thorac Dis 2018; 10: 2908-15.

12) Anyanwu AC, Chikwe J, Adams DH. Tricuspid valve repair for treatment and prevention of secondary tricuspid regurgitation in patients undergoing mitral valve surgery. Curr Cardiol Rep 2008; 10: 110-7.
13) Chan V, Burwash IG, Lam BK, et al. Clinical and echocardiographic impact of functional tricuspid regurgitation repair at the time of mitral valve replacement. Ann Thorac Surg 2009; 88: 1209-15.

14) Fukunaga N, Okada Y, Konishi Y, et al. Late outcome of tricuspid annuloplasty using a flexible band/ring for functional tricuspid regurgitation. Circ J 2015; 79: 1299-306.

15) Angeloni $E$, Melina $G$, Benedetto $U$, et al. Impact of prosthesis-patient mismatch on tricuspid valve regurgitation and pulmonary hypertension following mitral valve replacement. Int J Cardiol 2013; 168: 4150-4.

16) Grapsa J, Dawson D, Pandis D, et al. Mitral valve repair results in better right ventricular remodelling than valve replacement for degenerative mitral regurgitation: a three-dimensional echocardiographic study. Hellenic J Cardiol 2012; 53: 279-86.

17) Hata H, Fujita T, Miura S, et al. Long-term outcomes of suture vs. Ring tricuspid annuloplasty for functional tricuspid regurgitation. Circ J 2017; 81: 1432-8.

18) Gürsoy M, Bakuy V, Hatemi AC, et al. Long-term prognosis of mild functional tricuspid regurgitation after mitral valve replacement. Anadolu Kardiyol Derg 2014; 14: 34-9.

19) Benedetto U, Melina G, Angeloni E, et al. Prophylactic tricuspid annuloplasty in patients with dilated tricuspid annulus undergoing mitral valve surgery. J Thorac Cardiovasc Surg 2012; 143: 632-8.

20) Chikwe J, Itagaki S, Anyanwu A, et al. Impact of concomitant tricuspid annuloplasty on tricuspid regurgitation, right ventricular function, and pulmonary artery hypertension after repair of mitral valve prolapse. J Am Coll Cardiol 2015; 65: 1931-8.

21) Ren WJ, Zhang BG, Liu JS, et al. Outcomes of tricuspid annuloplasty with and without prosthetic rings: a retrospective follow-up study. J Cardiothorac Surg 2015; 10: 81 .

22) Huang $X, G u C$, Men $X$, et al. Repair of functional tricuspid regurgitation: comparison between suture annuloplasty and rings annuloplasty. Ann Thorac Surg 2014; 97: 1286-92. 